\title{
Lemierre's syndrome: an often forgotten but potentially life-threatening disease
}

\author{
C Lee *, Lorraine HY Sinn, Sonia HY Lam, WM Lam
}

Hong Kong Med J 2016;22:184.e1-2

DOI: $10.12809 / \mathrm{hkmj} 154696$

A 20-year-old Chinese female with good past health presented to the emergency department in October 2014 with a few days history of fever, dizziness, and headache. She was hypotensive upon presentation with blood pressure of $73 / 48 \mathrm{~mm} \mathrm{Hg}$ and pulse rate of 90 beats/min but responded to fluid resuscitation. Physical examination revealed no other significant findings. Her white cell count was $4.4 \times 10^{9} / \mathrm{L}$ on admission but increased to $13.4 \times 10^{9}$ five days later. Neutrophil predominance was observed. Subsequent gradual decline and normalisation of the white cell count was noted after initiation of intravenous antibiotics.

Computed tomographic (CT) abdomen and pelvis was initially requested based on the clinical suspicion of intra-abdominal sepsis or gynaecological pathologies but yielded no remarkable findings. Chest $\mathrm{X}$-ray (CXR) was also performed for preliminary assessment and was initially unremarkable. Followup serial CXRs, however, showed an increasing number of cavitary lesions in both lungs with no zonal predominance (Fig 1).

Non-contrast CT thorax was deemed necessary

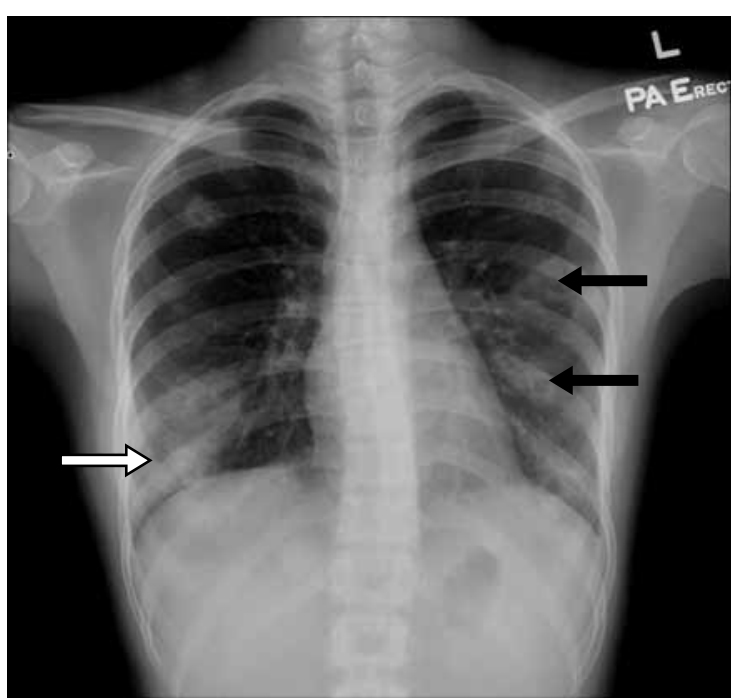

FIG I. Chest X-ray revealing multiple cavitary lesions of variable size in the bilateral lung fields with no zonal predominance (black arrows)

Area of consolidation is also evident in the right lower zone (white arrow) for further characterisation of these lesions and confirmed multiple cavitary lesions of variable sizes involving all the lung lobes (Fig 2). Thick irregular walls with fluid content and perifocal consolidative changes were noted in some of these lesions, which are compatible with an infective process. Further imaging workup was then arranged to identify any potential source of the septic emboli but echocardiogram did not reveal any heart valve vegetation.

On further questioning, the patient reported mild left neck pain. Doppler ultrasound of the neck was then performed based on the clinical suspicion of Lemierre's syndrome. A markedly narrowed lumen with thickened wall and dampened flow signal was noted across the left internal jugular vein suspicious of venous thrombosis (Fig 3). This was subsequently confirmed on contrast CT neck and thorax (Fig 4).

Mycoplasma-Fusobacterium polymerase chain reaction study confirmed the causative organism as Fusobacterium necrophorum, which is the classic bacteria described in Lemierre's syndrome.

The patient was promptly treated with intravenous antibiotics and anticoagulant. Her

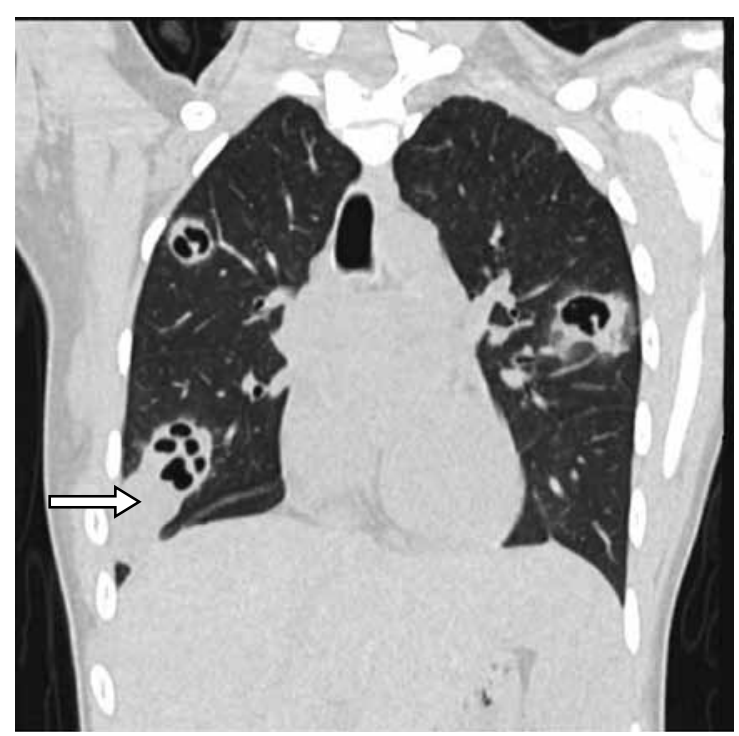

FIG 2. Computed tomographic thorax demonstrating multiple cavitary lesions of variable size involving all the lung lobes

The right lower zone lesion shows perifocal consolidative changes (white arrow) in some of these lesions, compatible with an infective process 


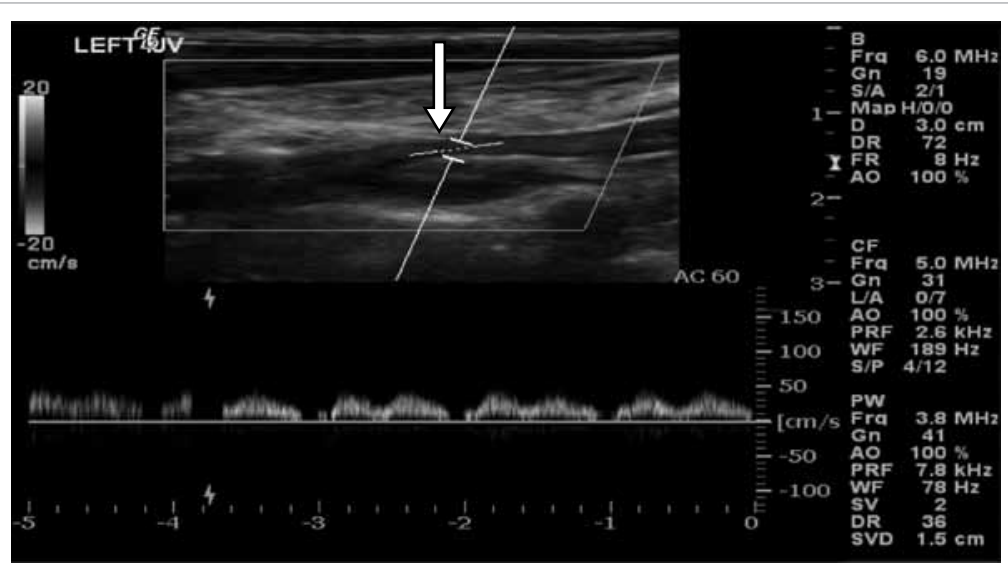

FIG 3. Markedly narrowed lumen (white arrow) with thickened wall and dampened flow signal is noted across the left internal jugular vein suspicious of venous thrombosis

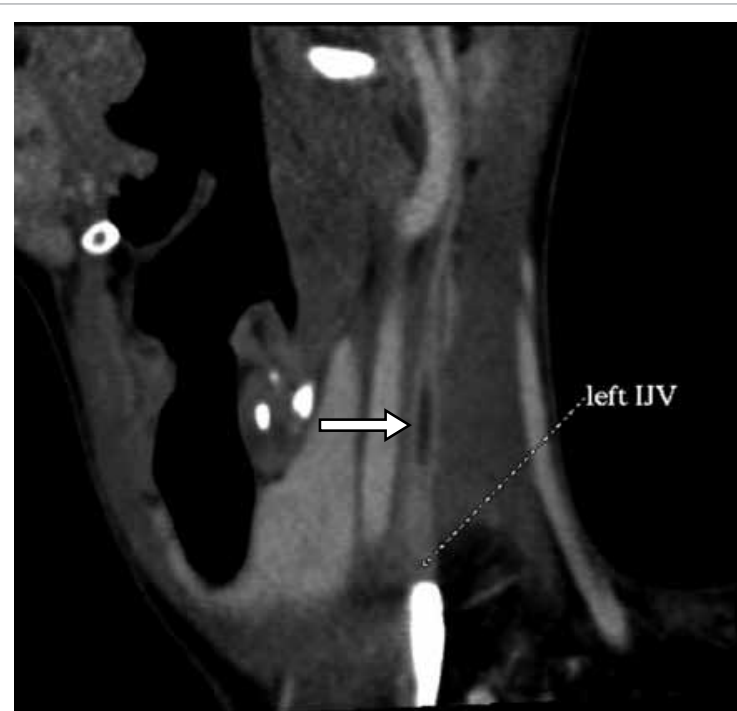

FIG 4. Reformatted computed tomographic image in an oblique sagittal projection showing a filling defect in the mid portion of the left internal jugular vein (IJV) [white arrow], compatible with jugular venous thrombosis

clinical condition gradually improved and followup CT scan demonstrated interval resolution of the cavitary lung lesions. She was subsequently discharged with oral antibiotics and anticoagulants.

Lemierre's syndrome remains a rare yet potentially life-threatening disease especially in young adults. ${ }^{1} \mathrm{~A}$ high index of clinical suspicion is therefore imperative to ensure prompt and timely imaging investigations that are integral to its diagnosis. Unrecognised and untreated systemic dissemination can result in a poor prognosis. ${ }^{2}$ Contrast-enhanced CT played a pivotal role in terms of evaluation of the pulmonary sepsis and assessment of the jugular vein thrombosis in this patient. ${ }^{3}$

An earlier version of this paper was presented as a poster at the European Society of Thoracic Radiology held in Barcelona, Spain on 4-6 June 2015.
C Lee *, MB, BS, FRCR

LHY Sinn, MB, BS, FRCR

SHY Lam, MB, BS, FRCR

WM Lam, MB, BS, FRCR

Department of Radiology, Queen Mary Hospital, Pokfulam, Hong Kong

* Corresponding author: leechunbruce@gmail.com

\section{References}

1. Shook J, Trigger C. Lemierre's Syndrome. West J Emerg Med 2014;15:125-6.

2. Lai C, Vummidi DR. Images in clinical medicine. Lemierre's Syndrome. N Engl J Med 2004;350:e14.

3. Screaton NJ, Ravenel JG, Lehner PJ, Heitzman ER, Flower CD. Lemierre syndrome: forgotten but not extinct-report of four cases. Radiology 1999;213:369-74. 